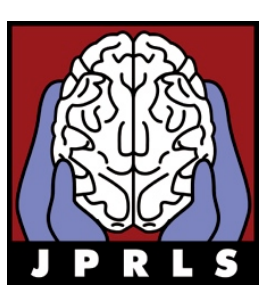

Open Access via www.jprls.org

Journal of Parkinsonism \& Restless Legs Syndrome

\title{
Pain in Lower Extremities in a Patient with Spina Bifida and Spinal Dysraphism
}

\author{
Iftekharul Haq,* Mohammed A. Rana \\ Department of Neurosurgery, Thunder Bay Regional Hospital, Lakehead University Thunder Bay, Ontario, Canada
}

\section{HISTORY}

A 55 year old right handed male presented to the neurosurgical service with chief complaint of aching pain in both calves. The pain was located in his shins below the knees. The pain occurred late in the evenings and increased before going to bed. His pain interfered with falling asleep, however, once he was asleep there was no pain. In the daytime, he had no pain in the lower extremities. These symptoms started about three years ago and gradually worsened. He denied any weakness, sensory numbness, tingling, bowel-bladder problems, or gait difficulty. He could walk unlimited distances.

The patient was a known case of diastematomyelia with a history of spina bifida from childhood. He also had cervical and thoracic surgery performed for spinal dysraphism. He was not on any medications.

He was married and living with his wife and had three children. He did not smoke. He used to drink alcohol only socially and worked as an optometrist. Family history was significant for diabetes and high blood pressure.

\section{Physical EXamination}

Upon examination, his speech was clear and fluent. Higher mental functions were intact. Examination of the cranial nerves was normal. Motor

* Correspondence to: Iftekharul Haq, Department of Neurosurgery, Thunder Bay Regional Hospital, Lakehead University, Thunder Bay, Ontario, Canada. Email: iftikharul_haq@hotmail.com. testing showed normal bulk, tone, and strength. There was no resting tremor. Finger-nose-finger and heelto-shin testing were normal. Deep tendon reflexes were $2+$ and symmetrical. Plantar reflexes were flexor. Primary sensory modalities were normal. Romberg test was negative. He was able to touch his thighs and extension of the lumbosacral spine was 20 degrees and lateral flexion was 20 degrees bilaterally. Straight leg raising test was normal on both sides. He was able to walk on tiptoes and heels. Tandem gait was also normal.

A differential diagnosis of symptoms secondary to spina bifida, spinal dysraphism, and diastematomyelia or polyneuropathy, as well as degenerative spine disease was considered.

\section{INVESTIGATIONS}

MRI of the spine did not show any compression of the exiting nerve root or thecal sac, which was initially thought to be the cause of his symptoms.

NCS/EMG did not show any evidence of peripheral nerve dysfunction, radiculopathy, plexopathy, or peripheral nerve entrapment.

\section{MANAGEMENT}

A neurology consultation was requested. The neurologist entertained the differential diagnosis of restless legs syndrome (RLS). Serum iron profile and $\mathrm{BUN} / \mathrm{Cr}$ were requested, but were normal. Patient was started on $0.25 \mathrm{mg}$ pramipexole, taken 45 minutes before bed. This was increased to $0.5 \mathrm{mg}$ after one week. Three days later, the patient started to notice 
partial improvement of his symptoms, and two weeks later reported almost complete relief.

Although various spinal abnormalities have been reported to cause symptoms similar to RLS, this patient's constellation of symptoms was consistent with the RLS, presenting with the sort pain which has been reported in the literature. ${ }^{2}$

\section{REFERENCES}

1. Hartmann M, Pfister R, Pfadenhauer K. Restless legs syndrome associated with spinal cord lesions. J Neurol Neurosurg Psychiatry 1999; 66(5): 688-9.

2. Hornyak M, Stiasny-Kolster K, Evers S, Happe S. Restless legs syndrome and nocturnal leg pain: Differential diagnosis and treatment. Sleep Med 2011; 12(2): 186-9. 